\title{
Biomonitoring equivalents: A screening approach for interpreting biomonitoring results from a public health risk perspective
}

\author{
S.M. Hays ${ }^{\text {a,* }}$, R.A. Becker ${ }^{\text {b }}$, H.W. Leung ${ }^{\text {c }}$, L.L. Aylward ${ }^{\text {d }}$, D.W. Pyatt ${ }^{\text {e }}$ \\ a Summit Toxicology, L.L.P., Allenspark, CO, USA \\ b American Chemistry Council, Arlington, VA, USA \\ ${ }^{\mathrm{c}}$ Independent Consultant, Danbury, CT, SA \\ d Summit Toxicology, L.L.P., Falls Church, VA, USA \\ ${ }^{\mathrm{e}}$ Summit Toxicology, L.L.P., Lafayette, CO, USA
}

Received 12 June 2006

Available online 6 October 2006

\begin{abstract}
Advances in both sensitivity and specificity of analytical chemistry have made it possible to quantify substances in human biological specimens, such as blood, urine, and breast milk, in specimen volumes that are practical for collection from individuals. Research laboratories led by the Centers for Disease Control and Prevention (CDC) in its series National Report on Human Exposure to Environmental Chemicals [Centers for Disease Control and Prevention (CDC), 2005. Third National Report on Human Exposure to Environmental Chemicals. NCEH Pub. No. 05-0570.] are dedicating substantial resources to designing and conducting human biomonitoring studies and compiling biomonitoring data for the general population. However, the ability to quantitatively interpret the results of human biomonitoring in the context of a health risk assessment currently lags behind the analytical chemist's ability to make such measurements. The traditional paradigm for human health risk assessment of environmental chemicals involves comparing estimated daily doses to health-based criteria for acceptable, safe, or tolerable daily intakes (for example, reference doses [RfDs], tolerable daily intakes [TDIs], or minimal risk levels [MRLs]) to assess whether estimated doses exceed such health screening levels. However, biomonitoring efforts result in measured chemical concentrations in biological specimens (the result of absorption, distribution, metabolism and excretion of administered doses) rather than estimated intake doses. Quantitative benchmarks of acceptable or safe concentrations in biological specimens (analogous to RfDs, TDIs, or MRLs) needed to interpret these levels exist for very few chemicals of environmental interest. This paper discusses issues inherent in converting existing health screening benchmarks based on intake doses to screening levels for evaluating biomonitoring data, and presents methods and approaches that can be used to derive such screening levels (termed "Biomonitoring Equivalents," or BEs) for a range of chemicals and biological media.
\end{abstract}

(C) 2006 Elsevier Inc. All rights reserved.

Keywords: Biomonitoring; Biomarkers; Risk assessment; Pharmacokinetics; Exposure reconstruction; Dosimetry; PBPK

\section{Introduction}

Exposure assessment is an integral part of the classical risk assessment process. The objective of exposure assessment is the quantification of the magnitude, duration, frequency and routes of exposure (e.g., air, water, food, soil, etc.) to chemicals, as well as the characterization and enu-

\footnotetext{
* Corresponding author. Fax: +1 3037472086.

E-mail address: shays@summittoxicology.com (S.M. Hays).
}

meration of the exposed populations. Conventional exposure assessments begin with estimation or measurement of exposure point concentrations and assumptions regarding media contact or intake rates for the demographic groups under consideration to yield a value of applied dose (units of $\mathrm{mg} / \mathrm{kg} / \mathrm{day}$ ). Exposures (in the appropriate units) are compared to screening toxicity criteria such as reference doses or concentrations (RfDs and RfCs), minimal risk levels (MRLs), tolerable daily intakes (TDIs), or a Unit Cancer Risk (UCR) to evaluate whether exposures exceed such criteria. 
Advances in analytical chemistry, in combination with a recognition of the substantial uncertainties involved in estimating environmental exposures, have resulted in a shift toward assessing humans' exposures to chemicals through biomonitoring, defined here as measuring the concentration of chemicals or their metabolites in blood, urine, breast milk, hair and other biological samples. Biomonitoring has the potential to decrease the uncertainty inherent in estimating exposures by conventional exposure assessment methods and to provide a more biologically relevant measure of true exposure. Conventional environmental exposure assessments typically incorporate conservative assumptions designed to provide upper bound estimates of possible intake rates, but the accuracy of the estimates can often not be determined, and in many cases they overestimate actual exposures, sometimes by orders of magnitude (Gosselin et al., 2006; Ewers et al., 1996, 2004). Biomonitoring can provide a direct measure of an individual's exposure and can integrate exposures from multiple pathways and sources, although it cannot, by itself, identify the specific sources or pathways of exposures or the relative contributions from multiple sources. Because biomonitoring is an indicator of internal dose, biomonitoring can also provide exposure estimates that are more directly related to the concentration of the active agent at the target site or organ, a key determinant of toxicity and/or pharmacological response, than estimated intakes or measures of concentration of a chemical in soil, water, or air. For these reasons, biomonitoring is fast becoming the "gold standard" of environmental exposure assessment (Needham et al., 1999).

The reasons for conducting biomonitoring studies fall within several broad categories. As articulated by the $\mathrm{CDC}$, these are to (a) determine which chemicals get into members of the general population and at what concentrations, (b) determine the prevalence of people with levels above known toxicity levels, (c) establish reference ranges, (d) assess the effectiveness of public health efforts to reduce exposure, (e) determine if exposure levels are higher in some groups than in others, (e) track temporal trends in levels of exposure, and (f) set priorities for research on human health effects (CDC, 2005).

Under specific conditions, biomonitoring data can be used to meet one of the most critical objectives of risk assessment - to determine whether individuals or a population are at an increased risk of experiencing adverse health effects associated with an exposure to a specific substance. Criteria for the evaluation of biomonitoring data in a health risk context have a substantial history in the occupational setting. In the United States, the American Conference of Governmental Industrial Hygienists (ACGIH) began developing biomonitoring-based reference values known as biological exposure indices $\left(\mathrm{BEIs}^{\circledR}\right)$ in the early 1980s (Fiserova-Bergerova, 1987). The ACGIH defines the BEIs ${ }^{\circledR}$ as representing the levels of determinants that are most likely to be observed in specimens collected from a healthy worker exposed to chemicals following inhalation exposure at the Threshold Limit Value $\left(T_{L V}{ }^{\circledR}\right)$. Outside the United States, the Deutsche Forschungsgemeinschaft (DFG, 2005) in Germany has also developed biological monitoring reference values called biological tolerance values (BATs), and the World Health Organization (WHO, 2005) maintains similar values referred to as biomonitoring action levels (BALs). BEIs ${ }^{\circledR}$, BATs and BALs all refer specifically to occupational populations and exposure scenarios only.

In contrast, for the general population, health-based screening levels for biomonitoring data exist for very few chemical substances (notable exceptions being lead, mercury, arsenic, cadmium, and ethanol). Epidemiologic studies attempting to correlate biomonitoring data with measured biochemical changes or health effects are unlikely to provide definitive screening levels for many chemicals in the short or long term due to relatively small sample sizes, complicated temporal relationships between detection of a chemical measured in human subjects and occurrence of a biochemical response or manifestation of a health effect, and potential alternative causes that may confound apparent exposure-response relationships. However, population and individual screening risk assessments could be made by comparing measured biomonitoring levels in individuals or populations to existing screening criteria such as RfDs, MRLs, or TDIs. This comparison cannot be conducted directly because almost all regulatory health-based toxicity screening criteria are based on an intake level $(\mathrm{mg} / \mathrm{kg} /$ day) or a concentration in an environmental medium (air, water, soil, etc.) which corresponds to an acceptable level of intake. However, the substantial effort already invested in developing these screening exposure guidelines can be leveraged through translation of these guidelines into biomonitoring equivalents ("BEs") as a basis for interpreting biomonitoring results for specific chemicals in a health risk context.

This paper describes several methods that can be used to calculate chemical- and biological media-specific concentrations, or BEs, equivalent to these existing screening criteria (for example, a $\mathrm{BE}_{\mathrm{RfD}}$ would provide a biomonitoring level equivalent to the RfD; a $\mathrm{BE}_{\mathrm{MRL}}$ would correspond to the MRL, etc.) for many chemicals of environmental interest. Since the presence of a chemical in a biological tissue or fluid indicates that exposure to the chemical has occurred, but does not necessarily imply the presence or magnitude of health risks, the BE terminology itself does not imply "safety" or "risk." Rather it refers explicitly back to the existing screening criteria that underlie the specific value. This paper also discusses issues and uncertainties related to the development and use of BEs. In particular, the process and approaches described here will result in screening values of varying reliability and robustness. The reliability of the derived BEs will depend on the available database of pharmacokinetic data for each chemical, the proximity of the sampled biological tissue or medium to the critical target tissue, understanding of population variability, and other factors discussed in detail below. For these reasons, 
BEs can be regarded as interim screening tools that can be used to identify chemicals with low margins of safety as candidates for additional detailed epidemiologic or exposure pathway evaluation. Finally, considerations for the design of biomonitoring studies to maximize their utility for interpretation of biomonitoring data from a public health risk perspective are discussed.

\section{Considerations and approaches for deriving biomonitoring equivalents}

\subsection{Pharmacokinetics as a determinant of biological media concentrations}

Ambient levels of chemicals in various media (air, water, food, and soil) do not always correlate with uptake into the human body, or, more importantly, with the concentration of a compound (parent compound or a metabolite of the parent compound) in the target tissue. Consequently, one major use of biomonitoring is to define more accurately the amount of chemical that has crossed physiological barriers to enter the body internally (Henderson et al., 1989). Linking applied dose/exposure to measurements of concentration of the parent compound or metabolite in a biological medium requires pharmacokinetics, which describes the rate processes of absorption, distribution, metabolism, and elimination of a chemical in the human body (Fig. 1). There are several types of models that can be used to describe and predict the pharmacokinetics of chemicals in animals and humans, but regardless of the model used, there are inherent principles of pharmacokinetics that should be taken into account when attempting to interpret biomonitoring data from a health risk perspective.
Regardless of the pharmacokinetic approach used, some assumptions about an exposure scenario must be made to convert a regulatory exposure guideline (such as an RfD) to a biomonitoring equivalent (BE). Such regulatory exposure guidelines are often presented in terms of chronic daily doses, implying a sort of steady-state exposure regimen, but in reality constant exposures to chemicals in the environment do not occur. If a chemical is found in food, for example, exposure occurs only during discrete events when food with residues of that chemical is consumed. If a substance is in drinking water, exposure occurs only when water is consumed or other exposure to water occurs (e.g., showering). Exposure to residues in consumer products is similarly intermittent. Exposures to chemicals in the air have the greatest potential to be constant, but substantial variations in air concentrations can occur among indoor and outdoor air, in a car, at the workplace, etc. Therefore, actual human exposures to environmental chemicals can be at the same time both discrete and intermittent.

The elimination behavior of many chemicals can be approximated using a one-compartment, first-order open model description of the kinetics. The average or steadystate concentration for a chemical is equivalent to:

$C_{\text {ss_avg }}=\frac{\mathrm{ADD} * t_{1 / 2}}{V * \ln (2)}$

where $C_{\text {ss avg }}$ is the steady-state (average) concentration, ADD is the average daily dose, $V$ is the volume of distribution, and $t_{1 / 2}$ is the half-life for elimination. The steadystate concentration is independent of the frequency of exposures, as long as the ADD remains the same. For instance, the average steady-state concentration will be the same for a chemical that someone is exposed to once, twice

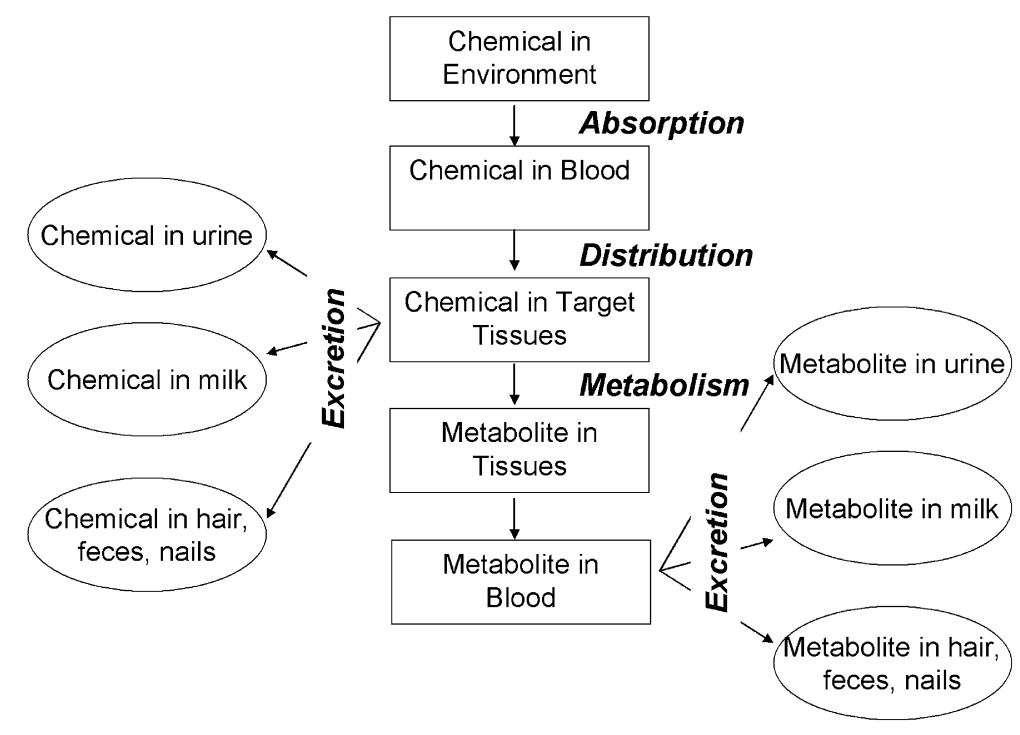

Fig. 1. The pharmacokinetic properties of a chemical control the relationship between administered dose or external exposure level and measured concentrations in biological media including blood, urine, feces, milk, hair, nails, etc. Even a simplistic understanding of these relationships can provide the basis for deriving a screening biomonitoring equivalent (BE) to an external exposure regulatory guideline such as an RfD. 
or 10 times per day, as long as the total daily dose is the same (see, for example, Fig. 2a and b). The variation or oscillation between the maximum and minimum concentrations, though, even at steady state, are dependent upon the frequency of exposure and half-life of the chemical, even while maintaining the same total daily dose (Fig. 2a and b). There will be no fluctuations if exposure is constant all day and night long (constant infusion, for example). Oscillations will increase if the total daily dose is divided into a few discrete exposure events in a day (e.g., each time a person takes a drink of water or has a meal). The largest rise and fall will occur if the total daily dose is experienced in a single event. The potential degree of daily fluctuation around the chronic steady-state average concentration is dependent upon the half-life of elimination, with larger oscillations occurring for chemicals with the shortest halflife for elimination (Fig. 3).

Therefore, when calculating a BE for a chemical corresponding to an oral dose (e.g., RfD, TDI, or any other health-based criteria that is expressed in units of mass/ body weight/day), it may be most informative to report both the steady-state (average) predicted concentration as well as the maximum daily concentration in the medium of interest predicted assuming chronic daily exposure to one event per day amounting to the entire daily dose. Since the once-daily exposure scenario will yield the maximum predicted concentration levels consistent with the regulatory screening level (and may correspond directly with the animal data underlying the RfD if it is based on a gavage dosing regimen) it represents an upper bound on the biomonitoring equivalent level that is consistent with the regulatory guidance exposure levels. These temporal variations could result in a false conclusion that portions of the populations were experiencing exposures above the RfD if only the average concentration is reported. This issue is most important for compounds with relatively rapid elimination behavior (elimination half-lives of a couple of days or less), and should be considered in designing studies and interpreting biomonitoring data.
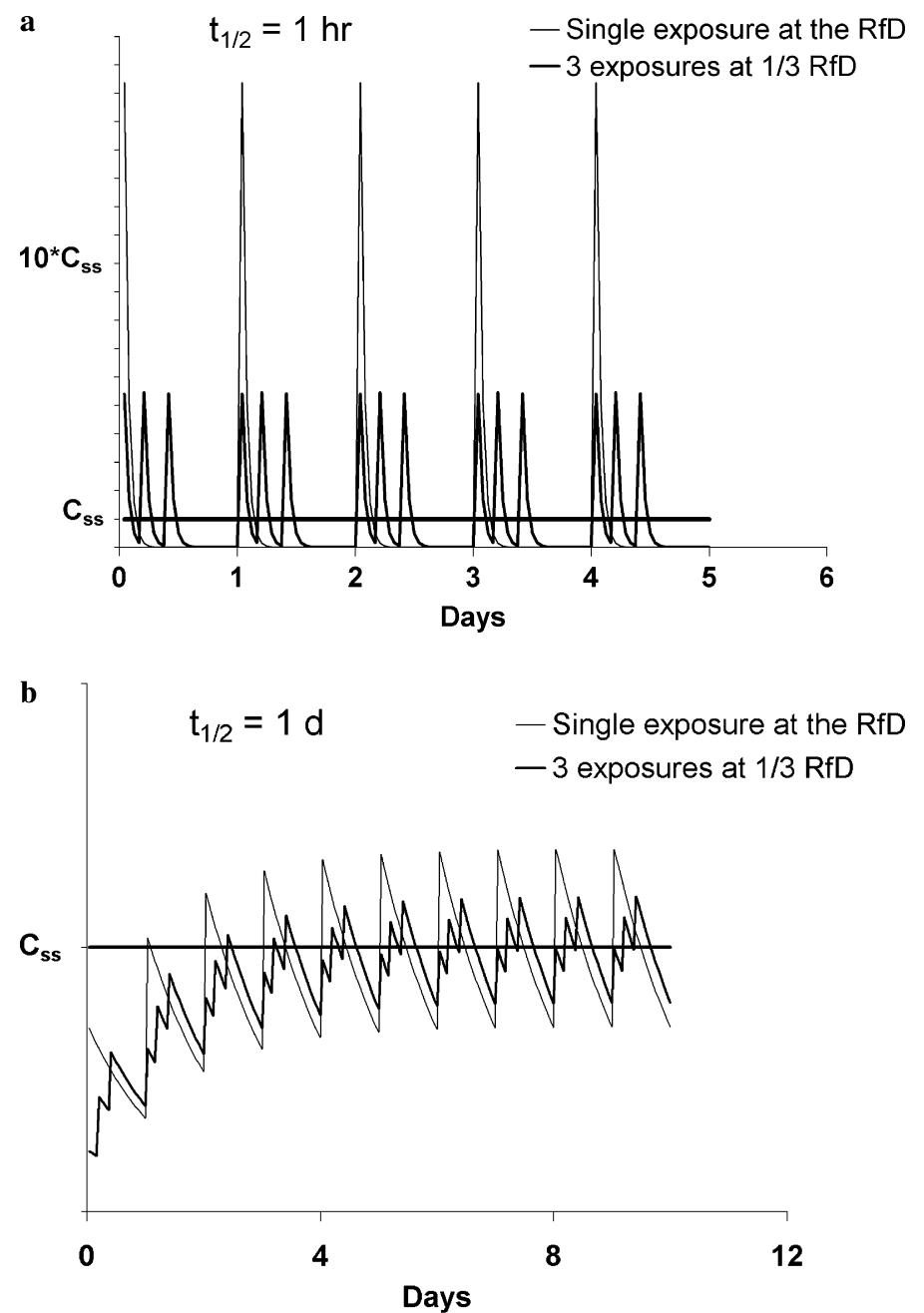

Fig. 2. Schematics demonstrate the relationship between dosing regimen and half-life of elimination in predicting the variation in concentrations that could be observed in biological media assuming first-order kinetics. When the half-life for elimination is much shorter than the dosing interval, the potential variations in concentrations observed may be very large. 


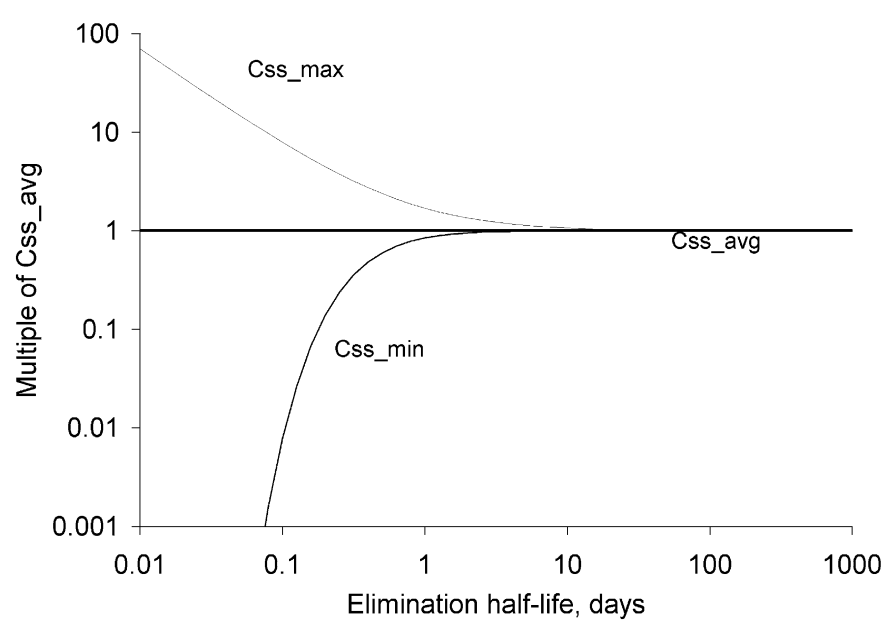

Fig. 3. Theoretical potential range of daily fluctuations in blood levels as a result of once-daily chronic exposure to a chemical as a function of the elimination half-life of the chemical assuming first-order kinetics. For chemicals with relatively short half-lives of elimination, the range of daily oscillations in concentration $\left(C_{\mathrm{ss} \min }\right.$ to $\left.C_{\mathrm{ss}} \max \right)$ can be very wide compared to the theoretical average steady state concentration $\left(C_{\mathrm{ss} \text { avg }}\right)$ arising from constant infusion. Random sampling in a theoretical population exposed daily to identical doses could produce a wide range of observed blood levels simply due to this potential oscillation.

\subsection{Forward (direct) dosimetry versus reverse (reconstructive) dosimetry approaches}

As discussed above, the pharmacokinetic properties of a chemical dictate the relationship between external and internal exposures, and pharmacokinetics provide the basic tools for interpreting biomonitoring data in the context of existing external exposure screening criteria. However, there are two ways to approach this problem:

- Begin with the external exposure criteria of interest (for example, an RfD) and then use pharmacokinetic knowledge to derive the expected biomonitoring concentration or range of concentrations of a substance in the biological medium of interest (forward approach); or

- Use pharmacokinetics to back-extrapolate from a measured biomonitoring concentration to a range of plausible exposures or applied doses and compare this range of derived applied doses with the external exposure screening criteria of interest (reverse approach).

Each of these approaches, which we term the forward or reverse dosimetry approach, respectively, has advantages and disadvantages. Ultimately, the choice between the two approaches will be dictated by the objectives of a given effort. This manuscript is focused on detailing methods that can be used to apply currently available, intake-based toxicity screening criteria to interpret human biomonitoring data in a health risk context while at the same time introducing the least amount of uncertainty.

Both forward and reverse dosimetry approaches require pharmacokinetic modeling. The reverse dosimetry approach has the advantage that the result of the modeling will yield an applied dose that can then be readily compared to existing regulatory guidance values (e.g., RfDs, RfCs, UCRs, etc.). The major disadvantage is that this modeling approach requires an inverse solution for a system with numerous variables in which there is no unique solution (Rigas et al., 2001; Sohn et al., 2004) (i.e., there are, within limits, any number of exposure scenarios that could yield the same biomonitoring level). In starting with a population-based distribution of biomonitoring levels (i.e., a range of biomonitoring levels), reverse dosimetry requires back-calculating to a population distribution of applied doses, yielding a wide range of potential exposures associated with the observed distribution in biomonitoring data. Researchers have attempted to back-calculate exposures from biomonitoring data, with discouraging results due to the variability in potential exposure scenarios and human pharmacokinetics (see for example Rigas et al., 2001; Sohn et al., 2004).

Nevertheless, the reverse dosimetry approach can be effectively used under some conditions. The utility of the reverse dosimetry approach will depend upon a solid understanding of likely exposure scenarios or development and use of accepted standardized exposure scenarios for specified populations, taking into account variability. Monte Carlo methods could be used, in conjunction with distributions of exposure parameters, to yield distributions of applied doses for specified populations given an observed distribution of biomonitoring data.

The forward dosimetry approach, in which a model is used to calculate the biomonitoring equivalent to being exposed at the regulatory guidance value, alleviates some of the challenges posed by the reverse dosimetry method. For instance, the model produces a single biomonitoring equivalent value starting from a single exposure level, with only one unique solution. Even if variability in pharmacokinetic behavior is incorporated into the model, the range of variability in resulting predicted tissue concentrations is smaller than the range of solutions yielded by the reverse dosimetry approach (Fig. 4). The other advantage is that using the forward approach will yield an internal dose (e.g., concentration of the parent compound or metabolite in blood) corresponding to the external dose criterion of interest. Using an "internal dose" has been recognized as a distinct advantage in the environmental toxicology field (NRC, 1994, etc.). Therefore, calculating a biomonitoring equivalent for a regulatory exposure criterion is moving in the direction of providing a screening "internal dose measure" associated with an external dose criterion for each chemical being evaluated.

Key characteristics of any approach used to screen biomonitoring data in a health risk context should be; (1) the approach should reduce or minimize uncertainty in the risk assessment paradigm, and (2) the approach should be relatively easy to communicate. In the remainder of this paper, we focus on the forward dosimetry approach for screening biomonitoring data in a health risk context. 

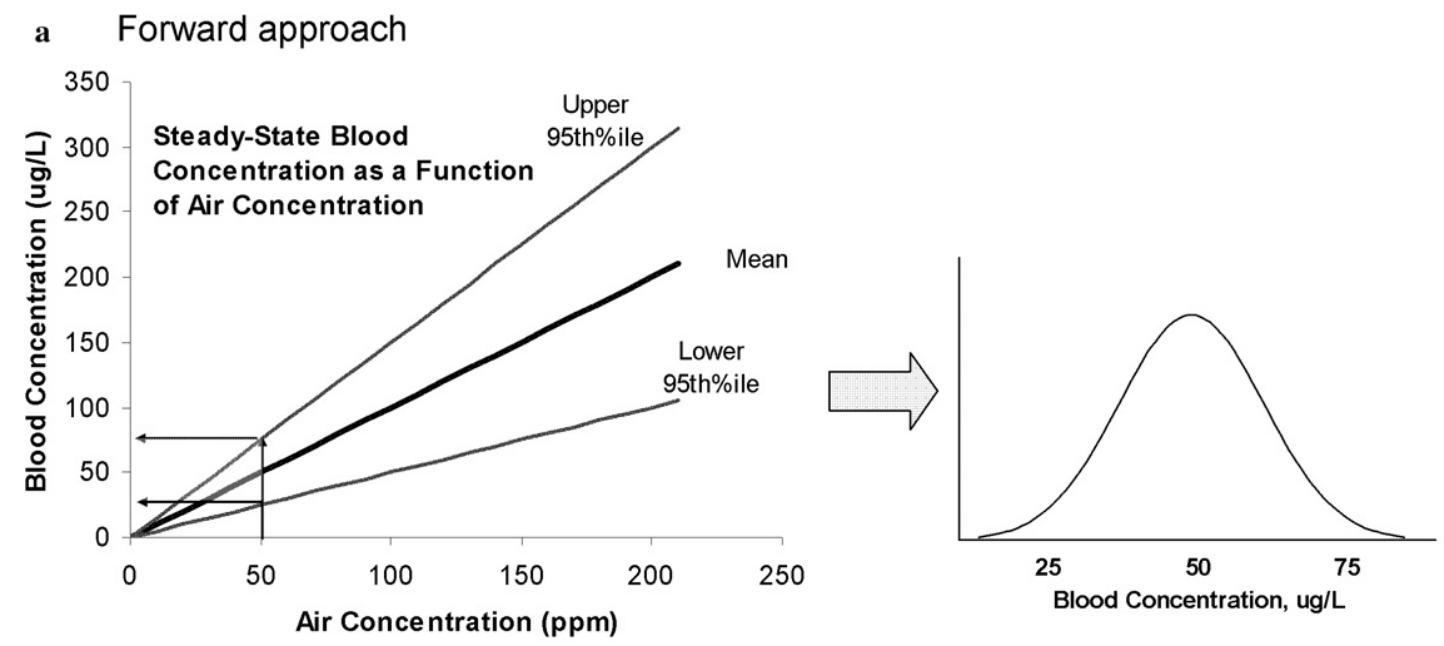

b Reverse approach
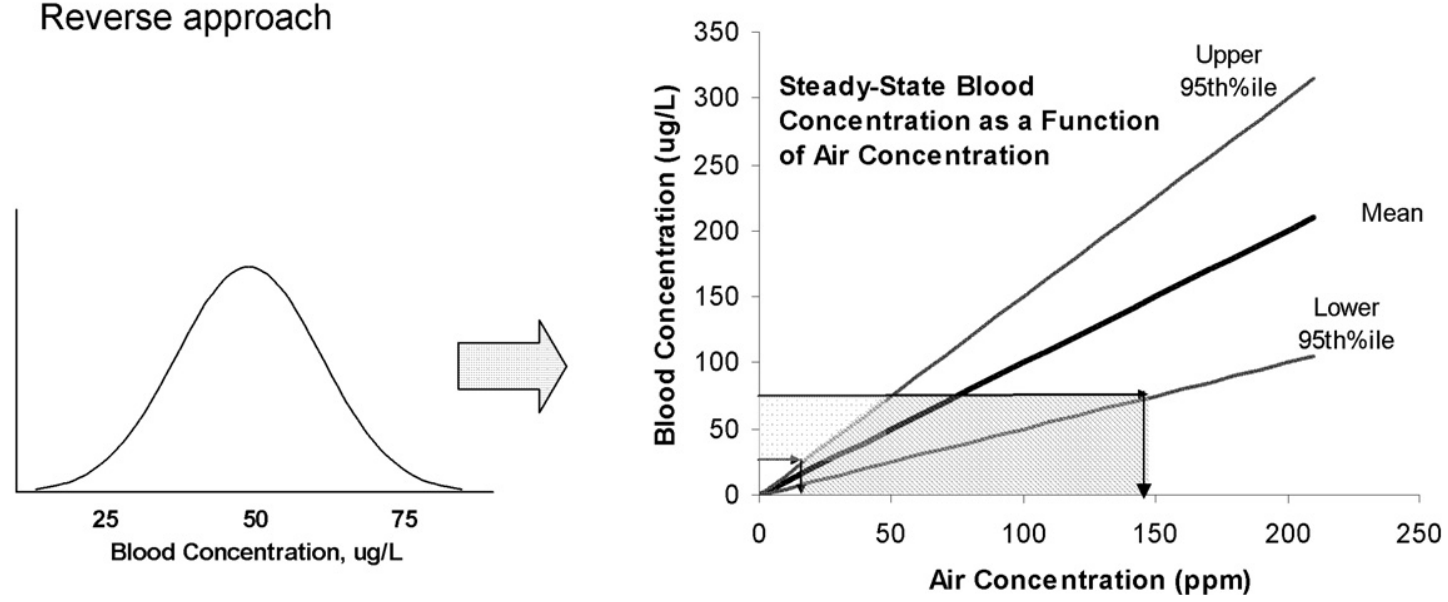

Fig. 4. Forward vs. reverse approach. Population variations in kinetic behavior lead to a range of blood concentrations associated with a given air concentration at steady state. Using the forward dosimetry approach (Part a), an RfC of $50 \mathrm{ppm}$ could be estimated to correspond to a mean steady-state blood concentration of $50 \mu \mathrm{g} / \mathrm{L}$ with a $95 \%$ C.I. of 25 to $75 \mu \mathrm{g} / \mathrm{L}$. However, using the reverse dosimetry approach (Part b), a wider range of air concentrations ( 20 to about $150 \mathrm{ppm}$ ) could be associated with the same observed range of blood concentrations due to the variations introduced by kinetic variability. The forward approach identifies the range of blood concentrations consistent with exposures at the RfC.

\subsection{Methods for deriving BEs}

There are numerous methods based in the discipline of pharmacokinetics available for relating external exposures to a corresponding level of parent compound or metabolite in a biological specimen (e.g., blood, urine, exhaled air, tissue, breast milk, hair, nails, etc.); they vary in terms of complexity, scientific rigor and required effort. As with any public health evaluation, the confidence in a calculated BE will be dependent upon the thoroughness of the evaluation. Some evaluations can be easily developed by using simple extrapolation procedures, and the BEs calculated using a simple approach may be considered a first approximation or screening level assessment. On the other hand, some evaluations may be very sophisticated and involve substantial effort to develop complex and well validated quantitative models (such as PBPK models). BEs developed using a more thoroughly evaluated method might be considered fully "validated" and be more universally applicable to a wide range of scenarios. However, for chemicals with relatively simple and well-understood pharmacokinetic properties, simpler approaches may be just as valid.

None of these methods address potential shortcomings in the existing regulatory exposure guidelines; they simply provide methods to estimate concentrations in biological media that are consistent with exposure at these external exposure guidelines.

Some of the methods that can be used to develop BEs and reviewed in this manuscript include:

1. Extrapolation from occupationally derived biomarker levels such as the Biological Exposure Indices (BEIs) set by the ACGIH

2. Human PK studies and one-compartment steady-state models

3. Multi-compartment and PBPK models

4. Animal PK studies 
Each of these methods is discussed in some detail below. In general, the methods outlined below focus on blood as the medium of interest, although similar approaches can be used to convert to BEs for use with other sampled media. Interpretation of data for each medium requires consideration of issues specific to that medium. For example, urine is frequently sampled for metabolites from exposures to a wide range of drugs and chemicals, and appropriate methods for standardization of urinary output volumes must be considered.

The approaches outlined here are predicated on several assumptions:

- That internal measures of dose are superior to external measures as predictors of health effects. This might not be true, for example, if a chemical causes a local toxic or irritant effect in the lung above a certain air concentration.

- That the regulatory guidelines (e.g., RfCs, RfDs, MRLs, etc.) used as the starting point for derivations of BEs are reasonable and protective. Nothing in the BE approaches outlined here will address underlying deficiencies in existing regulatory guidelines. Similarly, when several differing regulatory guidelines exist for the same chemical, BEs can be derived associated with each of them, but will not resolve the underlying differences among the existing criteria.

- That the sampled medium and analyte provide information relevant to prediction of potential toxicity. The sampled medium (or the measured analyte), while providing an internal estimate of dose, may have been chosen on the basis of ease of collection or measurement rather than ease of interpretation in the context of potential toxic responses. The more distant the sampled medium and measured analyte are from the target organ and active agent, the more difficult it may be to accurately interpret the data in a health risk context.

- That the appropriate exposure regimen to assume for extrapolation from external to internal dose is chronic, steady-state exposure at the regulatory guideline. The approaches outlined below generally derive BEs associated with repeated, ongoing exposure scenarios, not episodic exposures (except to the extent that daily fluctuations due to variations in timing of daily exposure are addressed).

\subsubsection{Extrapolation from biomonitoring standards established for occupational settings}

The industrial hygiene community has been calculating BEs for over 20 years (Fiserova-Bergerova, 1987). The Biological Exposure Index $\left(\mathrm{BEI}^{\circledR}\right)$ is the ACGIH's version of the $\mathrm{BE}$ and is defined as being the biological levels of the determinant in biological specimens collected from a healthy reference man $(170 \mathrm{~cm}, 70 \mathrm{~kg}$, about $12 \%$ body fat) occupationally exposed ( $8 \mathrm{~h}$ per day, 5 days per week) to a chemical or a specified mixture while performing work with a moderate energy expenditure $(50 \mathrm{~W}$, pulmonary ventilation about $20 \mathrm{~L} / \mathrm{min}$ ) (Fiserova-Bergerova, 1990). ACGIH BEIs ${ }^{\circledR}$ are typically derived using one of two approaches: developing correlations between exposure concentrations (usually at occupationally relevant exposures) and a BEI ${ }^{\circledR}$ using human exposure data, or using a pharmacokinetic model to calculate a $\mathrm{BEI}^{\circledR}$ that would correspond with exposures at the threshold limit value $\left(\mathrm{TLV}^{\circledR}\right)$ (Leung and Paustenbach, 1988). Calculating a BEI $^{\circledR}$ for an occupational exposure incorporates different assumptions than typically used for a general population exposure because of the discrete and non-continuous exposures $(8 \mathrm{~h} /$ day, 5 days per week) experienced in the workplace. Because of this, a BEI ${ }^{\circledR}$ is usually derived to correspond with a specific workday and time of day (e.g., at the end of the fifth workday, the morning after returning from the weekend, etc.). Despite the fact that BEIs ${ }^{\circledR}$ are derived for non-steady-state exposures, BEIs ${ }^{\circledR}$ can be used to inform the derivation of BEs for the general population. Linear scaling (or simple pharmacokinetic modeling for chemicals with rapid elimination profiles) can be used to reduce the $\mathrm{BEI}^{\circledR}{ }^{\circledR}$ to correspond to continuous, 7 day per week exposures when exposures are to the more long-lived compounds. Then this adjusted BEI ${ }^{\circledR}$ may be further modified to correspond to the regulatory guideline of interest (for example, an RfC) which is likely to be more stringent than the TLV ${ }^{\circledR}$. The ACGIH has established BEIs ${ }^{\circledR}$ for 42 compounds. BALs, BATs, and other biological indices of exposure corresponding to workplace exposure limits have been established for numerous additional compounds and could serve as the starting point for additional derivations using this approach (DFG, 2005; WHO, 2005; Lauwerys and Hoet, 2001). Consideration needs to be made for potential routes of exposures. Work place exposures are often dermal and respiratory, while non-occupational exposures, especially to non-volatiles, may be primarily through the diet.

\subsubsection{Human exposure PK studies and one-compartment steady-state models}

For chemicals without existing BEIs ${ }^{\circledR}$ (or similar occupational biomonitoring standards), the easiest method available for developing the relationship between exposures and concentrations in biological medium is with actual human exposure data from studies that measured an actual biological media concentration of interest following controlled exposures in human volunteers. There are numerous studies reporting pharmacokinetic data in humans, especially from studies conducted before the 1980s. These studies were usually conducted at exposure levels that were, at the time, close to or equivalent to applicable occupational exposure limits. The types of compounds that have the most human exposure data of this type are volatile organics and some pesticides.

With this method, a simple relationship can usually be drawn between the exposure concentration (or oral dose) and the concentration of compound in the biological medium in question. The most difficult part of using this 
method is determining the shape of the relationship between external exposure and the concentration in the biological medium of interest. Usually only a few exposure concentrations were studied, and usually these were at concentrations much greater than current regulatory exposure guidelines for the general population (e.g., RfC, RfD, etc.). Extrapolating to lower exposure concentrations comes with some degree of uncertainty, but a linear relationship between exposure concentration and steady-state BE is usually a reasonable assumption, unless information exists to suggest the exposure experiments were done at sufficiently high concentrations to saturate metabolic processes.

Data from human exposure studies is most useful when inhalation of relatively volatile chemicals is evaluated because steady-state is usually achieved relatively rapidly, at least for compound levels in blood. Empirical relationships between inhaled air and blood concentrations at steady-state have been published (Andersen, 1981). These equations seem to be best suited for volatile organics that are metabolized in the liver, and can be used to predict blood concentrations following inhalation exposures.

Human exposure studies reported in the literature following a single oral dose requires additional extrapolations to predict steady-state concentrations in blood or urine. However, considerable insight has been gained from the pharmaceutical arena which is applicable to setting oral BEs. Classical relationships between AUC, peak concentrations, elimination rates, and steady-state concentrations in first-order systems are well understood (Gibaldi and Perrier, 1982) and can be used to analyze such data sets.

Fig. 5 shows the typical blood profile of a generic chemical following a single oral dose. The concentration of a chemical will increase in the blood stream until a peak level is achieved when the rate of absorption matches the rate of elimination. After the peak concentration has been achieved, the rate of decline in the concentration of the chemical will be dictated by the rates of excretion and/or metabolism. In pharmacokinetic studies in which human volunteers were administered a single oral dose of a chemical, the reported AUC, peak concentration, and/or halflife for elimination following a single dose can be used to derive the predicted steady-state concentration following continuous dosing at the same dosing level (mg/day). These classical first-order relationships can be used without too much error for an oral administration pharmacokinetic study if the rate of absorption is rapid compared to the rate of elimination (i.e, the peak concentration is reached at a time that is less than half of one half-life). This is usually achieved for most chemicals in which the half-life of elimination is longer than about $2 \mathrm{~h}$.

A similar approach involves the use of a one-compartment model that accounts for all tissues that act as a reservoir for a chemical in the body (apparent volume of distribution). The mathematical formulation of the onecompartment model under steady-state exposure conditions results in solutions that are similar to those arising from simple first-order kinetics described above and in Fig. 5. However, single compartment models may also include explicit factors to account for bioavailability of administered doses, urinary excretion rates, and other simple features affecting distribution and elimination. This type of model can work well for oral exposures to chemicals with reasonably well-understood distribution and elimination characteristics. Many of the parameters required for a one-compartment model are readily available from

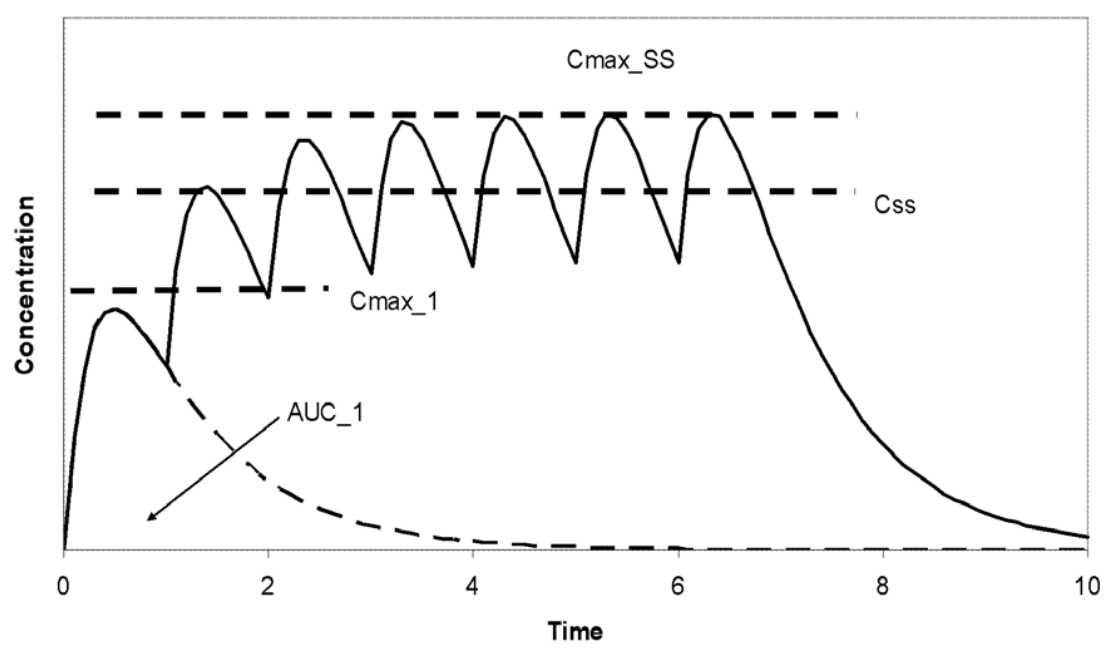

Fig. 5. Data gained from a single oral dose in humans or animals can be used to predict the accumulation and elimination behavior of a chemical under steady-state conditions. The curve above corresponds to measured serum concentrations of a generic chemical after a single oral dose. The steady-state concentrations (average and maximum) can be related to the measured parameters from the single oral dose using the following equations (Gibaldi and Perrier, 1982): $C_{\mathrm{ss}}=\frac{\mathrm{AUC} \_1}{\tau}$, and $C_{\max \_s s}=\frac{C_{\max -1}}{\left(1-\mathrm{e}_{-\tau \operatorname{th}(2) / t_{1 / 2}}\right)}$, where $C_{\mathrm{ss}}$ is the predicted average steady-state concentration, AUC_1 is the measured area under the concentration curve following a single oral dose, $C_{\max \_s s}$ is the predicted maximum serum concentration achieved after repeated (steady state) exposures, $C_{\max 1}$ is the measured maximum serum concentration following a single oral dose, $\tau$ is the dosing interval, and $t_{1 / 2}$ is the measured half-life of elimination. Notice that $C_{\max \_s s}$ is dependent upon dosing interval, while $C_{\mathrm{ss}}$ is not. 
various sources. For instance, the Agency for Toxic Substances Disease Registry (ATSDR) Toxicological Profiles (www.atsdr.cdc.gov) usually compile information on halflife, bioavailability and volume of distribution or clearance, and also discuss more complex pharmacokinetic models when they are available.

\subsubsection{Multi-compartment and physiologically-based pharmacokinetic (PBPK) models}

In the simplest case, the pharmacokinetics can be described by a single exponential term dependent on elimination. The chemical is assumed to achieve instantaneous distribution within a single homogeneous compartment. However, physiologically, it is often more appropriate to regard the body as representing a simple two-compartment open system in which the distribution to certain peripheral tissues is not an instantaneous process. In such a system, the chemical initially enters a central compartment, which represents blood and various richly perfused tissues in which distribution is rapid, and is subsequently distributed (more slowly) to a second peripheral compartment. Elimination occurs from the central compartment, so that the chemical in the peripheral compartment must transfer back into the central compartment to be eliminated.

At steady-state, chemical concentrations in both the central (blood) and peripheral compartments can be estimated using equations similar to those used for one-compartment first-order systems. These relationships can be used to predict steady-state blood concentrations associated with repeated intakes at a regulatory guideline level.

Physiologically based pharmacokinetic (PBPK) models are multi-compartment $\mathrm{PK}$ models in which compartments represent actual tissue masses and organs and the flow of chemical between compartments is described using blood flow to inter-connect the compartments in an anatomically correct relationship (Fig. 6). Unlike simple one and twocompartment PK models, the description of chemical disposition is governed by anatomically correct compartments and physiologically correct blood flow distributions. Multiple PBPK models can also be linked together to describe the disposition of the parent compound and multiple metabolite(s). This quality can be particularly advantageous for chemicals in which a metabolite is the preferred biomonitoring target. Besides being able to quantitatively describe chemical/metabolite concentrations in target organs and blood, PBPK models have also been used to quantitatively account for chemical/metabolite elimination from exhaled air, urine, milk, and feces and to model factors including enzyme induction and potential interactions between chemicals. In this respect, PBPK models are multidimensional and may prove the most valuable and robust of the various tools available to interpret biomonitoring data by integrating numerous analytes and medium of concern. PBPK models have been successfully employed to describe the kinetics of a wide range of drugs, metals and chemicals. Table 1 provides a list of environmental chemicals for which at least one PBPK model has been devel-

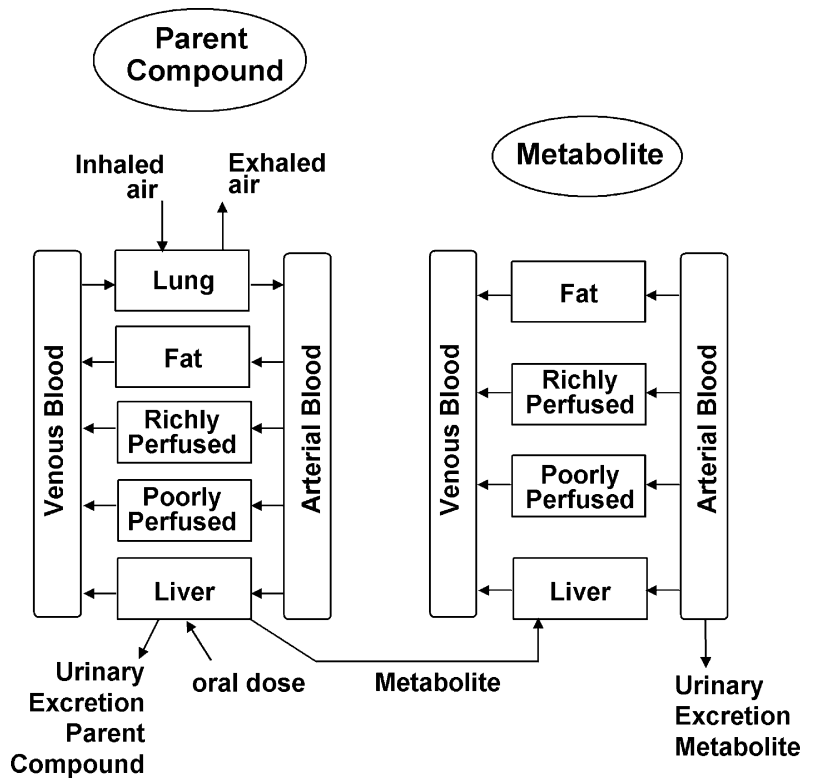

Fig. 6. Schematic of a PBPK model linking parent compound and metabolite pharmacokinetics. A PBPK model can be used to predicted concentrations in various biological media following multiple exposure from multiple routes or following complicated exposure regimens.

oped. PBPK models have been successfully employed to derive BEIs ${ }^{\circledR}$ for occupational exposures for a wide range of compounds (Leung, 1992; Droz et al., 1989; Thomas et al., 1996; Truchon et al., 2006).

When human models do not exist, a first-approximation can be developed using a PBPK model that has been parameterized for an experimental animal species provided that the animal species is the same one used in the critical study on which the regulatory standard is based (discussed further in the next section).

\subsubsection{Animal pharmacokinetic studies}

Often, the pharmacokinetics of a chemical have been determined in animal toxicology studies, even when no corresponding data exists for humans. For instance, the National Toxicology Program (NTP) performs pharmacokinetic studies that coincide with their bioassays. The data obtained in animals can be useful for estimating a BE for humans using several approaches (Fig. 7).

If the pharmacokinetics of the chemical in question are available in the animals from the critical study chosen to set the RfC, RfD, etc., the rodent BE from the NOAEL or LOAEL group (that is, the concentration of chemical in the rodent blood or other sampled medium) can be used as a starting point. Then the human BE can be obtained by dividing the animal $\mathrm{BE}$ by appropriate uncertainty factors. If this approach is used, some UFs typically used in setting an RfD (to extrapolate from animals to humans and to account for interindividual variability among humans) may not be required to produce an equally protective $\mathrm{BE}$. This issue is discussed in more detail below.

The other potential approach is to use measured pharmacokinetic relationships in laboratory animals from 
Table 1

Chemicals with published PBPK models as of 2005

\begin{tabular}{lll}
\hline - Acetone & - Ethanol & - Methylene chloride \\
- Acrylonitrile & - 2-Ethoxyethanol & - MTBE \\
- Arsenic & - Ethyl acrylate & - Napthalene \\
- Benzene & - Ethyl acetate & - Nickel \\
- Benzo[a]pyrene & - Ethylene dibromide & - Nicotine \\
- Bromobenzene & - Ethylene oxide & - Physostigmine \\
- Butadiene & - Furan & - PCBs \\
- 2-Butoxyethanol & - Hexabromobiphenyl & - Soman \\
- Cadmium & - Hexachlorobenzene & - Styrene \\
- Carbon tetrachloride & - Hexane & - Toluene \\
- Chlorfenvinphos & - Isoamyl alcohol & - Tetrachloroethane \\
- Chloroalkanes & - Isofenphos & - Tetrachloroethene \\
- Chloroethanes & - Kepone & - Tetrahydrofuran \\
- Chloroform & - Lead & - Trichloroethane \\
- Chloropentafluorobenzen & - Melphalan & - Trichloroethene \\
- Chromium & - Mercury & - Trichloro- \\
& & trifluoroethane \\
- DDT & - Methanol & - Vinyl acetate \\
- Dichlorobenzene & - 2-Methoxyethanol & - Vinyl chloride \\
- Dichloroethane & - Methotrexate & - Vinylidene chloride \\
- Dichloroethene & - Methylmercury & - Vinylidene fluoride \\
- 1,4-dioxane & - Methylethylketone & - Xylenes \\
- Dioxin & & - Zinc \\
\hline
\end{tabular}

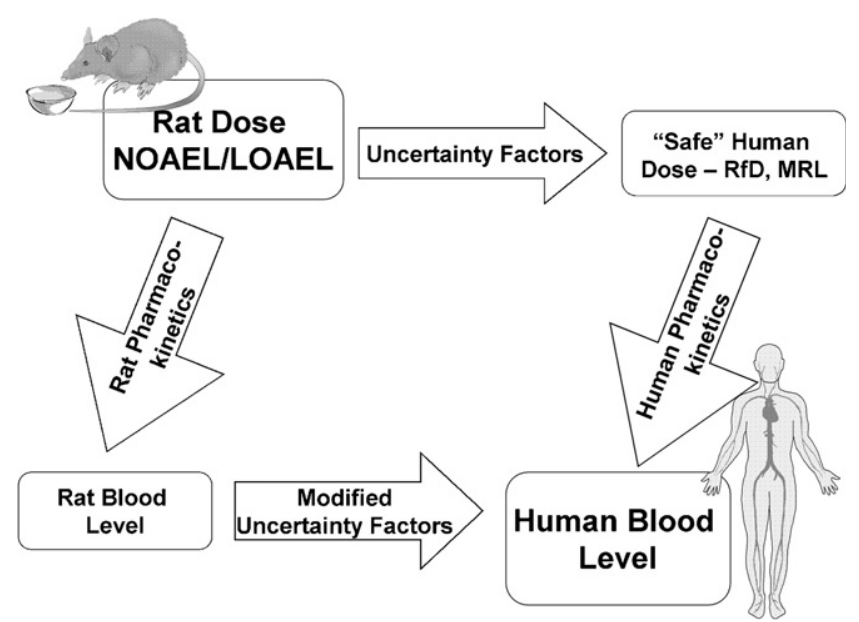

Fig. 7. Laboratory-derived NOAELs and LOAELs can be converted to BEs for human blood or other media concentrations through at least two pathways.

studies not directly used in the derivation of the regulatory guideline and use known species allometric scaling factors to calculate a human equivalent BE. Using this approach, a BE measured in an animal species that is not part of the critical study, and most likely at a dose different from the NOAEL or LOAEL from the critical study, is used as a starting point, and several extrapolations are needed. The first extrapolation will need to be to the animal species from the critical study and to the dose used as the point of departure for the regulatory guidance (NOAEL, LOAEL, etc.). Then, the adjusted animal $\mathrm{BE}$ should be divided by the composite UF and finally scaled to a human equivalent $\mathrm{BE}$ using known species allometric scaling factors. Kirman et al. (2003) derived interspecies allometric scaling factors for several species and for a wide range of compound-specific issues, such as inhalation versus oral exposures and whether the critical compound is the parent compound or a metabolite. These types of allometric scaling factors should be consulted for deriving BEs using this approach.

\section{Interpretation issues}

\subsection{Using estimated BEs to focus public health evaluations and data development}

The BEs corresponding to existing health-based screening guidelines, developed using the types of methods outlined above, clearly must be used with caution and with the understanding of their inherent limitations and uncertainties. However, even with these uncertainties and limitations recognized, such BEs could serve important public health purposes. In particular, the availability of BEs could provide a rationale for prioritizing further study. If preliminary biomonitoring data for a chemical show levels in the general population far below estimated BEs for the compound, this could be important information used to decide whether further biomonitoring of the substance is warranted or whether resources should be applied to evaluate another substance. Conversely, if preliminary data show biomonitoring levels at or above the estimated $\mathrm{BE}$, additional research to strengthen the biomonitoring database, evaluate human pharmacokinetics and dynamics for the compound, identify population exposure sources and pathways, and implement exposure reduction programs could be prioritized. In cases where the margin of exposure in general population biomonitoring (the derived BE divided by the observed biomonitoring concentration) raised some degree of concern, such substances might also be considered as the subject of epidemiologic or mechanistic studies to further clarify potential risks in the general population or for exposure studies to better understand relative source contributions. If data are insufficient to derive BEs corresponding to existing exposure guidelines, this could serve as impetus to fund research on human and/or animal pharmacokinetics so that observed biomonitoring results can be interpreted in a health risk context.

\subsection{Setting regulatory standards with biomonitoring in mind}

In the not too distant future, regulatory standards will likely be set with biomonitoring in mind so that biomonitoring levels can be more easily interpreted from a public health perspective. In the meantime, the methods and principles outlined in this manuscript can be used to convert current regulatory standards to BEs. However, there are some regulatory standards already in place that can serve as useful templates for how regulatory standards should be set in the future to allow easy interpretation of biomonitoring studies. For instance, the EPA has set an RfC for ethylene glycol monobutyl ether (EGBE; or 2-butoxyethanol) in which the 
critical dose measure in rats was determined to be the peak blood level of the primary metabolite of EGBE; 2-butoxyacetic acid (BAA (USEPA, 1999)). The critical toxic endpoint was changes in mean corpuscular volume. Using a PBPK model, the EPA back-calculated the steady-state exposures (and thus the RfC and RfD) required to achieve the same critical dose measure in humans. For biomonitoring purposes, the EPA could easily establish a BE based on BAA concentration in blood. The type of assessment conducted by EPA for EGBE could serve as a useful template for future regulatory standard setting programs who wish to not only provide guidance on external environment concentrations, but also provide guidance on interpretation of biomonitoring levels.

\subsection{Use of uncertainty factors}

In the process of deriving regulatory exposure guidelines from animal data, uncertainty factors (sometimes referred to as safety factors) are generally used to compensate for areas of uncertainty (Dourson, 1993; Dourson et al., 2002). Typically, the experimentally derived NOAEL or calculated benchmark dose (BMD) is divided by these uncertainty factors (UF) to develop health-based standards to gain greater assurance of human health protection. For example, an UF of 10 is often used to extrapolate from animals to humans and 10 to extrapolate to the most sensitive in the human population to establish an RfD or RfC. These uncertainty factors include consideration for toxicokinetic variation as well as variations in intrinsic sensitivity (between species and among humans). To the degree that a $\mathrm{BE}$ is determined for a medium that is closely related to the relevant internal dose, the use of a BE reduces the animal-to-human toxicokinetic extrapolation uncertainty. Similarly, the potential variation in kinetic behavior included in the interindividual uncertainty factor is directly expressed in measured tissue concentrations, and additional adjustment for interindividual variations in kinetics using uncertainty factors may not be warranted. Methods for adjustment of the uncertainty factors incorporated in the RfD or other regulatory guidelines in converting to BEs is an area for significant discussion and is beyond the scope of this manuscript. However, it is clear that the use of a $\mathrm{BE}$ in a public health interpretation manner has the potential to reduce some of the uncertainty that is usually included in the current process for deriving RfDs, RfCs and similar guidelines, and this may be reflected in modifications to the uncertainty factors applied to the animal data underlying the original guidelines.

\subsection{Population versus individual risks}

As biomonitoring continues to become more common and receive public attention, individuals may seek analysis of their blood, hair, or urine in an attempt to assess personal risks from exposure to environmental chemicals. Currently, such analyses are only performed in a routine way for blood lead levels in children. However, in the near future, individuals may seek these analyses for a wider range of chemicals, either through their physicians or independently from laboratories through web services similar to those that currently advertise clinical laboratory testing to individuals for more traditional biochemical parameters. While the use of BEs on a population basis in a public health risk assessment arena has the potential to reduce uncertainty that currently exists in the environmental risk assessment field and perhaps to focus public health priorities, appropriate application and interpretation of such benchmarks for individuals will be more problematic. In particular, physicians may be called upon to answer questions from patients regarding the meaning of results of such analyses.

Most physicians have not been trained in such interpretation and probably will not have a detailed appreciation of the risk assessment paradigms and assumptions used to derive intake-based criteria such as RfDs, much less the methods used to convert such values to corresponding BEs. Two factors preclude the use of RfDs or the BEs for interpretation of individual risks. First, RfDs and RfCs typically are derived with a wide margin of safety and EPA states that the range of values around an RfD is "perhaps an order of magnitude" (Dourson, 1993). That is, these values are not "bright lines" that distinguish safe levels from levels diagnostic of a health effect or risk. As such, a simple exceedence of an $\mathrm{RfD}$, or the corresponding $\mathrm{BE}$, does not necessarily imply that an exposure level associated with adverse effects has been experienced. Second, a measured level (assuming it is accurate) represents a concentration at a point in time, but factors important for interpretation include whether an exceedence is transient or continuing and the type of biological effect the RfC, RfD, etc. is meant to protect from occurring. Health-based screening criteria such as RfDs are based on a variety of health effects and biological responses that range in severity from mild responses or biological changes to clearly adverse events. Therefore, an understanding of the type of biological effect that might be associated with an exceedance of a $\mathrm{BE}$ and the magnitude of uncertainty factors that are incorporated into the derivation of such criteria would be helpful for a health practitioner to understand how to advise his/ her patients. However, such information is not readily available or easily communicated to either physicians or their patients. Given these considerations, physicians' interpretation of potential health risks for individuals should be limited to suggesting little cause for concern if the measured levels fall below the $\mathrm{BE}$ and to providing information about the typical distribution of measured values in the general population. In this respect, the efforts by the CDC (2005) to present the distribution of measured chemical levels in the general population is a valuable resource. The development of other resources to assist in the education of physicians and public health officials regarding these and other issues will be critical. 


\subsection{Study design issues and uncertainty and variability associated with biomarker interpretation and selection}

Interpreting biomonitoring data using the $\mathrm{BE}$ approach has distinct advantages. It also comes with some uncertainties and variabilities. For each pharmacokinetic process (e.g., absorption, distribution, metabolism, and excretion; see Fig. 1) there are uncertainties that are both species-specific and chemical-specific. There are also inherent variabilities within the human population for each process that are both process-dependent and chemical-dependent (a full discussion of this issue is important but beyond the scope of this paper). The further a biomarker (analyte and medium) gets away from either the critical dose measure or the external exposure that the guideline was based upon, the more steps in extrapolation (and thus uncertainty and variability) are required to calculate a BE and thus are introduced into how biomonitoring results can be interpreted from a health risk perspective. For this reason, blood will most likely be the gold standard for biomarker interpretation for most chemicals.

The pharmacokinetic and mechanistic considerations and understandings that are required to estimate BEs corresponding to current regulatory exposure guidelines also can inform the design, focus, and implementation of future biomonitoring studies. The factors listed below should be evaluated in selecting analytes and biological media for study and in structuring such studies. Furthermore, when evaluating biomonitoring datasets for interpretation, these same factors need to be evaluated and considered.

- Pharmacokinetics of study compounds. The elimination half-life of the compound of interest should fall into an intermediate range, with optimal half-lives ranging from a typical exposure interval of approximately one day to approximately one month. Compounds with half-lives much shorter than the expected exposure interval will demonstrate extremely large fluctuations in the concentration in biological media with short term peaks (see Fig. 2). This will result in a wide range of concentrations in the collected samples which will make interpretation more difficult. Similarly, if the half-life of elimination is far longer than the usual exposure interval, the chemical will demonstrate significant accumulation over time and biomonitoring data will be more indicative of long term or historical exposures than reflective of current exposure levels. Such information may be useful, but this issue must be taken into account during the interpretation of biomonitoring data for highly persistent compounds (e.g., dioxins; see Aylward and Hays, 2002, for further discussions).

- Selection of analyte and biological medium for analysis. Pharmacokinetic and mechanism of toxicity information should inform the selection of parent compound versus metabolite and biological media for analysis. If the metabolite is more stable than a rapidly-metabolized parent, or if the metabolite is directly linked to toxicity, it may be a more desirable choice for analysis. However, analysis of a metabolite is most useful when the metabolite is not common to other exposures. For example, phenol, hippuric acid and cresols are derived from dietary components and are excreted in urine, complicating the assessment of exposure to benzene and toluene by biomonitoring of these metabolites. Similarly, urinary measurement of alkyl phosphates cannot distinguish between ingestion of the alkyl phosphates and the production of alkyl phosphates from in vivo metabolism of organophosphate pesticides (Duggan et al., 2003). Similarly, choice of biological medium for analysis will vary depending on practical considerations (invasiveness) and relevance to the target organ toxicity of interest. Relative ease of collection and relevance to target organ toxicity for a given media will vary by chemical and may not point to the same choice of biological medium for sampling, so the limitations inherent in the choices made in a given study should be acknowledged.

- Other study design considerations. Although not fully explored in this paper, the value of interpreting biomonitoring results from a public health risk perspective is dependent upon the design and quality of the underlying biomonitoring study. Biomonitoring studies must be designed with attention to a complex range of considerations, including appropriate analytical chemistry procedures (sample collection, storage, and analysis protocols), informed consent procedures, defined data quality objectives (including assessments of sample size and population representativeness) and a priori hypotheses to be tested with the data. A more complete discussion of these important issues is warranted but beyond the scope of this paper (see American Conference of Governmental Industrial Hygienists (ACGIH), 2001 for an expanded discussion of quality assurance issues for biomonitoring studies).

\section{Discussion}

Exposure assessment is an integral part of the health risk assessment process. Traditional exposure assessment methods often produce a highly uncertain dose (whether it is a single value or a range of doses) for a large population in which many assumptions have been made with respect to contact rates, frequency of exposures and rates of ingestion or inhalation. Such results may be overly simplistic because of the complexity of factors that influence the dose that has been absorbed into the body. To overcome these shortcomings, there has been increasing interest in biological monitoring techniques that measure the concentrations of chemicals in tissues and other biological fluids, thereby eliminating the need for making any assumptions about external exposure parameters. Several government agencies, notably the $\mathrm{CDC}$, have recently embarked on programs that monitor 


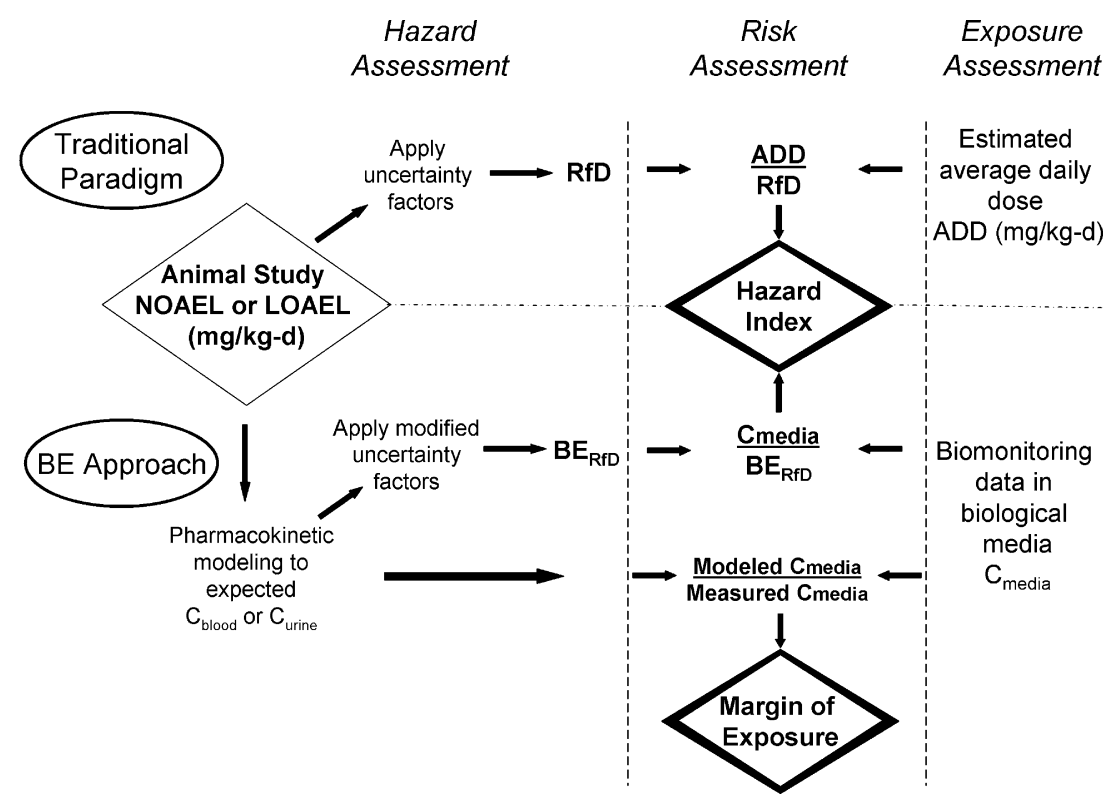

Fig. 8. The BE methodology can be used in ways analogous to the traditional risk assessment paradigm. Illustrated here is the process for non-cancer risk assessment using both the traditional hazard and exposure assessment approach and using a BE-based approach. The BE methodology can be used to estimate either a hazard index or a margin of exposure, depending upon the goal of the particular assessment, by comparing the measured concentration in a biological medium $\left(C_{\text {media }}\right)$ to the predicted concentrations in blood or urine ( $C_{\text {blood }}$ or $\left.C_{\text {urine }}\right)$ associated with the LOAEL, NOAEL, or RfD.

the levels of a wide range of chemicals in the general population (NHANES). However, scientifically derived reference guidelines are lacking for interpretation of biomonitoring data from a risk assessment perspective.

In the preceding sections we have demonstrated how screening BEs may be derived from existing exposure standards using a variety of pharmacokinetic approaches ranging from simple empirical extrapolation from human study data to more complex multi-compartmental models or full PBPK modeling. While the number of chemicals with validated PBPK models is somewhat limited, the recent focus of EPA on using PBPK methodologies for risk assessment will galvanize the research effort and broaden the list of chemicals with PBPK models and relevant PK data.

BEs can be used to assess biomonitoring results in the context of screening-level population health risk assessments, in a manner analogous to the way RfDs, RfCs and UCRs are currently used for estimated applied doses/external exposures (Fig. 8). The results of such evaluations can help to focus additional research, identify priorities (e.g., populations, chemicals, etc.) for further study and, importantly, provide a means to place biomonitoring levels in a health risk assessment context.

While screening BEs derived from traditional regulatory guidelines provide an initial approach to evaluating biomonitoring data, the BEs themselves have limitations and uncertainties that should be clearly understood.

- Unless the target tissue for toxicity is the sampled body medium, none of these measurements can be considered as true measures of effective or critical dose. However, they are likely to be improved surrogates over external dose estimates for most chemicals.
- The available pharmacokinetic data that can be used to convert from applied dose to concentrations in biological media vary widely from one chemical to another in quality, relevance, and robustness. Nonetheless, many of these pharmacokinetic relationships have been validated and exhibit predictive value for assessing the potential for toxic effects in a particular target organ or system. A clear description of the reliability and robustness of the underlying data should be presented with the BE derivation and incorporated in the process of assessing biomonitoring data against the BE. Such uncertainty is likely to be no greater than the uncertainties that typically are incorporated into the derivations of RfDs and similar criteria, however.

- BEs can be subject to uncertainty from physiological variations between individuals or over time in an individual. This variability may not be well understood across the population, particularly for subpopulations (for example, the children or the elderly) that may have not participated in pharmacokinetic studies. However, BEs are no more or less uncertain than the underlying regulatory exposure guidelines.

- BEs derived from existing regulatory exposure guidelines should be regarded as an interim step, bridging between the traditional risk assessment paradigm and more sophisticated understanding of the relationships between internal exposures and potential adverse effects for individual chemical substances.

The interpretation of biomonitoring data is and will remain a formidable challenge, even for those most closely involved in the science. Collectively, the medical and toxicology communities are a long way away from interpreting 
human biomonitoring data for all but a few environmental substances in a manner that can be used for medical diagnosis of an individual. However, developing reference standards such as BEs, from established toxicity criteria such as RfDs, RfCs, and UCRs, promises to provide a scientifically based approach for beginning to describe biomonitoring results in the context of population health risk assessments. The medical and toxicology communities have a responsibility to work with regulatory agencies to communicate to the public that presence of a chemical or its metabolite(s) in a biological tissue or fluid is regarded as evidence that exposure to the chemical has occurred, but it does not necessarily imply that any bodily harm has ensued or will result. Furthermore, the meaning of the reference limits (BEs) as screening benchmarks should be made perfectly clear so that there is no confusion what they represent. An important issue at hand is the communication of the current state of our knowledge base on the chemical of interest so that the uncertainties of the exposure assessment can be put into proper perspective.

\section{Acknowledgment}

This manuscript was funded in part by the American Chemistry Council.

\section{References}

American Conference of Governmental Industrial Hygienists (ACGIH), 2001. Introduction to the Biological Exposure Indices. ACGIH, Cincinnatti, OH.

Andersen, M.E., 1981. A physiologically based toxicokinetic description of the metabolism of inhaled gases and vapors: analysis at steady state. Toxicol. Appl. Pharmacol. 60, 509-526.

Aylward, L.L., Hays, S.M., 2002. Temporal trends in human TCDD body burden: decreases over three decades and implications for exposure levels. J. Expo. Anal. Environ. Epidemiol. 12, 319-328.

Centers for Disease Control and Prevention (CDC), 2005. Third National Report on Human Exposure to Environmental Chemicals. NCEH Pub. No. 05-0570.

Deutsche Forschungsgemeinschaft (DFG), 2005. MAK- and BAT-Values. Commission for the Investigation of Health Hazards of Chemical Compounds in the Work Area. VCH Publications, Weinheim, Germany.

Dourson, M. 1993. Modifying uncertainty factors for noncancer endpoints. Advanced Topics in Risk Assessment, Society of Toxicology 1993 Annual Meeting.

Dourson, M., Charnley, G., Scheuplein, R., 2002. Differential sensitivity of children and adults to chemical toxicity. II. Risk and regulation. Regul. Toxicol. Pharmacol. 35, 448-467.

Droz, P.O., Wu, M.M., Cumberland, W.G., Berode, M., 1989. Variability in biological monitoring of solvent exposure. I. Development of a population physiological model. Br. J. Ind. Med. 46, 447-460.

Duggan, A., Charnley, G., Chen, W., Chukwudebe, A., Hawk, R., Krieger, R.I., Ross, J., Yarborough, C., 2003. Di-alkyl phosphate biomonitoring data: assessing cumulative exposure to organophosphate pesticides. Regul. Toxicol. Pharmacol. 37, 382-395.

Ewers, U., Boening, D., Albrecht, J., Radel, R., Peter, G., Uthoff, T., 2004. Risk assessment of soil contamination in a residential area: the importance and role of human biological monitoring - a case report. Gesundheitswesen 66, 536-544.

Ewers, U., Wittsiepe, J., Schrey, P., Selenka, F., 1996. Levels of PCDD/ PCDF in blood fat as indices of the PCDD/PCDF body burden in humans. Toxicol. Lett. 88, 327-334.

Fiserova-Bergerova, V., 1987. Development of biological exposure indices (BEIs) and their implementation. Appl. Ind. Hyg. 2, 87-92.

Fiserova-Bergerova, V., 1990. Application of toxicokinetic models to establish biological exposure indicators. Ann. Occup. Hyg. 34, 639-651.

Gibaldi, M., Perrier, D., 1982. Pharmacokinetics: Second Edition, Revised and Expanded. Marcel Dekker, Inc., New York, New York 10016.

Gosselin, N.H., Brunet, R.C., Carrier, G., Bouchard, M., Feeley, M., 2006. Reconstruction of methylmercury intakes in indigenous populations from biomarker data. J. Expo. Sci. Environ. Epidemiol. 16, 19-29.

Henderson, R.F., Bechtold, W.E., Bond, J.A., Sun, J.D., 1989. The use of biological markers in toxicology. Crit. Rev. Toxicol. 20, 65-82.

Kirman, C.R., Sweeney, L.M., Meek, M.E., Gargas, M.L., 2003. Assessing the dose-dependency of allometric scaling performance using physiologically based pharmacokinetic modeling. Regul. Toxicol. Pharmacol. 38, 345-367.

Lauwerys, R., Hoet, P., 2001. Industrial Chemical Exposure: Guidelines for Biological Monitoring. CRC Press, LLC, Lewis Publishers, Boca Raton, FL.

Leung, H.W., 1992. Use of physiologically based pharmacokinetic models to establish biological exposure indexes. Am. Ind. Hyg. Assoc. J. 53, 369-374.

Leung, H.W., Paustenbach, D.J., 1988. Application of pharmacokinetics to derive biological exposure indexes from threshold limit values. Am. Ind. Hyg. Assoc. J. 49, 445-450.

National Research Council (NCR), 1994. Science and Judgment in Risk Assessment. The National Academies Press, Washington, DC.

Needham, L.L., Gerthoux, P.M., Patterson Jr., D.G., Brambilla, P., Smith, S.J., Sampson, E.J., Mocarelli, P., 1999. Exposure assessment: serum levels of TCDD in Seveso, Italy. Environ. Res. 80, S200-S206.

Rigas, M.L., Okino, M.S., Quackenboss, J.J., 2001. Use of a pharmacokinetic model to assess chlorpyrifos exposure and dose in children, based on urinary biomarker measurements. Toxicol. Sci. 61, 374-381.

Sohn, M.D., McKone, T.E., Blancato, J.N., 2004. Reconstructing population exposures from dose biomarkers: inhalation of trichloroethylene (TCE) as a case study. J. Expo. Anal. Environ. Epidemiol. 14, 204-213.

Thomas, R.S., Bigelow, P.L., Keefe, T.J., Yang, R.S., 1996. Variability in biological exposure indices using physiologically based pharmacokinetic modeling and Monte Carlo simulation. Am. Ind. Hyg. Assoc. J. $57,23-32$.

Truchon, G., Tardif, R., Droz, P.O., Charest-Tardif, G., Pierrehumbert, G., 2006. Biological exposure indicators: quantification of biological variability using toxicokinetic modeling. J. Occup. Environ. Hyg. 3, 137-143.

United States Environmental Protection Agency (USEPA), 1999. Toxicological Review of Ethylene Glycol Monobutyl Ether (EGBE). $<$ www.epa.gov/iris/toxreviews/0500-tr.pdf $>$.

World Health Organization (WHO), 2005. Biological monitoring of chemical exposure in the workplace guidelines. WHO Press, Geneva, Switzerland. 